\title{
Radially propagating turbulent structures in a linear helicon plasma device.
}

\author{
T. Windisch, O. Grulke*, and T. Klinger \\ MPI for Plasma Physics, EURATOM Association, D-17491 Greifswald, Germany
}

\begin{abstract}
The propagation properties of plasma density structures in a turbulent linear cylindrical helicon plasma are investigated. The governing instability is of the drift-wave type. An azimuthal limiter is used to steepen the radial density gradient to achieve a weakly developed turbulent state. The spatiotemporal evolution of turbulent plasma density fluctuations is investigated by Langmuir probe array measurements. In the strong plasma density gradient region the probability distribution function (PDF) of density fluctuations is rather symmetric. Here, the relict of a single $\mathrm{m}=1$ drift mode dominate the correlation of the fluctuations. Radially further outwards the fluctuations are dominated by large positive density bursts, associated with strongly asymmetric PDFs. The spatiotemporal measurements reveal that these intermittent bursts can be ascribed to radially propagating turbulent structures, which originate in the strong plasma gradient region. Their radial velocity reaches $5 \%$ of the local ion sound speed.
\end{abstract}

PACS:52.25.Fi, 52.25.Xz, 52.30.Ex, 52.35.Kt, 52.35.Ra

JNM keywords: Experimental Techniques, Diffusion

PSI-17 keywords: Turbulence

* Corresponding author address:Wendelsteinstr. 1, Greifswald, Germany

* Corresponding author E-mail: grulke@ipp.mpg.de

Presenting author: Olaf Grulke

Presenting author E-mail: grulke@ipp.mpg.de 


\section{INTRODUCTION}

It is a well know feature of plasma turbulence that the resulting transport is characterized by strong intermittency with the occurrence of sporadic large transport events. Such events can be ascribed to structures, which form in the turbulent region of the plasma and generally cause plasma transport by convection in potential vortices [1]. However, in recent years the direct radial propagation of turbulent structures across the confining magnetic field has been observed in tokamak scrapeoff layers (SOL) [2, 3]. These transport events reach relatively large radial velocities [4] and are thus of high importance for first wall erosion and recycling phenomena. Common models describing the radial propagation rely on magnetic field curvature, either by implying interchange mode dynamics [5] or polarization [6,7]. However, radial structure propagation has also been observed in linear magnetic field geometry without any magnetic field curvature $[8,9]$. Although the primary instability driving the turbulence remains under discussion, clear indications for radial propagation of turbulent density structures were found.

In this paper we present investigations of the propagation of turbulent density structures in the linear high-density helicon device VINETA. The governing instability was identified as being of the drift wave type $[10,11]$. They saturate usually into single coherent drift modes and can be driven to a weakly developed turbulent state. The paper is organized as follows: Sec. II outlines the experimental arrangement and the diagnostic approach. In Sec. III the main results of the temporal and spatiotemporal characteristics of turbulent fluctuations are presented before the major results are summarized in Sec. IV.

\section{EXPERIMENTAL SETUP}

The experiments were performed in the VINETA device [12]. It consists of a cylindrical stainless steel vacuum vessel (length $L=4.5 \mathrm{~m}$ ) immersed in a set of 36 magnetic field coils, which produce a homogeneous magnetic field of $B \leq 0.1 \mathrm{~T}$. The plasma production is via helicon wave heating [13] using a standard $m=1$ helicon antenna [14], which is placed around a glass tube vacuum extension (length $80 \mathrm{~cm}$, diameter $10 \mathrm{~cm}$ ) at one end of the device. The antenna is driven with RF of a fixed frequency of $f_{\mathrm{rf}}=13.56 \mathrm{MHz}$. With RF powers of $P_{\mathrm{rf}} \leq 3 \mathrm{~kW}$ peak, plasma densities of $n \leq 10^{18} \mathrm{~m}^{-3}$ at electron temperatures of $T_{e} \approx 3 \mathrm{eV}$ are achieved. The radial plasma profile size is mainly determined by the radial extent of the helicon source and the profile shape 
is generally a Gaussian. To decrease the radial density gradient scale length $L_{n}=|\nabla \log n|^{-1}$ an azimuthally symmetric limiter with radius $r_{a}=2 \mathrm{~cm}$ is introduced for the turbulence studies presented here. The limiter extends $20 \mathrm{~cm}$ along the magnetic field, which is larger than the mean free path $\lambda_{\mathrm{mfp}}$ for ionizing collisions with the Argon neutrals, which is $\lambda_{\mathrm{mfp}}=10 \mathrm{~cm}$ for the present parameters. The radial density profile behind this annulus downstream from the helicon antenna as measured with Langmuir probes is shown in Fig. 1. The peak plasma density in the center is with $n=4 \cdot 10^{17} \mathrm{~m}^{-3}$ slightly smaller than in operation without limiter, but develops strong radial gradients. The plasma potential profile is also peaked in the center. The azimuthal electron diamagnetic drift velocity exceeds the $E_{r} \times B$ drift in the central part of the plasma in magnitude, but is anti-parallel. However, in the plasma edge for $r>30 \mathrm{~mm}$ the $E_{r} \times B$ drift is larger. Relative density fluctuations are measured with negatively biased Langmuir probes assuming fluctuations of the ion saturation current to be proportional to plasma density fluctuations, $I_{i, s a t} \sim \tilde{n}$ (electron temperature fluctuations are neglected). For the spatiotemporal characterization of fluctuations a vertical array of 9 Langmuir probes (spacing $\Delta y=5 \mathrm{~mm}$ ) is used, which measures in an azimuthal cross-section downstream from the azimuthal limiter. The array is moved consecutively radially and the resulting two-dimensional domain covered with the probe array is $y=-20 \ldots 20 \mathrm{~mm}$ in vertical and $x=-120 \ldots 50 \mathrm{~mm}$ in horizontal direction relative to the plasma center at $x=$ $y=0 \mathrm{~mm}$. The data is analyzed using the conditional averaging technique $[15,16]$ to extract the coherent part of fluctuations. The reference signals for this conditional correlation analysis are density fluctuations at the radial position of maximum plasma density gradient ( $r=20 \mathrm{~mm}$ ) and in the edge plasma $(r=50 \mathrm{~mm})$, respectively.

\section{SPATIOTEMPORAL STRUCTURE DYNAMICS}

The temporal evolution of density fluctuations at three different radial positions $r=20,50,80 \mathrm{~mm}$ are depicted in Fig. 2. Shown are timeseries of density fluctuations normalized to their standard deviation and respective probability distribution functions (PDFs). At $r=20 \mathrm{~mm}$, i.e. at the position of maximum plasma density gradient, the maximum values of fluctuations reach $\approx 2 \sigma$. The corresponding PDF is symmetric and close to a Gaussian with skewness $s=0.2$ and kurtosis $k=-0.4$. Further out radially, the fluctuations become much more burst-like with positive large-amplitude distinct events. In the plasma edge at $r=50 \mathrm{~mm}$ the fluctuation level increases significantly, with maximum relative amplitudes of the burst events of $5 \sigma$. The PDF is more peaked and develops 
positive tails $(s=1.8, k=3.4)$. This characteristic is even more pronounced in the far plasma edge $(r=80 \mathrm{~mm})$. Here, the burst events dominate the fluctuations with maximum relative amplitudes of bursts reaching $8 \sigma$ in amplitude. The PDF is strongly peaked and highly asymmetric with long positive tails ( $s=2.7, k=10.1$ ). The absolute density amplitudes of the bursts correspond to the time-averaged density at $r \approx 20 \mathrm{~mm}$, which indicates that the origin of these bursts is in the density gradient region.

To get insight into the spatiotemporal dynamics of the turbulent fluctuations, conditionally averaged density fluctuations with the reference either positioned in the gradient region or in the plasma edge are measured in the azimuthal plane. For the density gradient reference case the results are shown in Fig. 3 for two different time lags $\tau$. The condition on the reference signal was an amplitude threshold and events are considered whose amplitudes exceed $0.5 \sigma$. The result of this procedure reveals that coherent fluctuations in this region are clearly dominated by a $m=1 \mathrm{drift}$ mode. The propagation of the mode is purely azimuthal in electron diamagnetic drift direction (counterclockwise in the representation of Fig. 3). The lifetime of the mode is longer than the time it takes for the mode to propagate around the plasma column.

The situation is different when the reference signal is taken in the plasma edge at $r=50 \mathrm{~mm}$. The conditionally averaged density fluctuations are shown in Fig. 4 for four different time lags spanning over $\tau=-16 \ldots 32 \mu \mathrm{s}$. The evolution of a monopolar positive amplitude structure is observed. The size of the conditionally averaged structure reflects the real structure size because it is dependent on the phase and amplitude of fluctuations, not only on the phase as in the case of the correlation size. The radial size of the structure is comparable to the spatial scale of the $\mathrm{m}=1$ mode structure, but it is azimuthally elongated. Its correlation time is longer than its typical eddyturnover time, identifying it as a coherent structure. Inspection of the temporal evolution of the structure reveals that its azimuthal propagation is opposite to the drift mode in $E_{r} \times B$ direction. Additionally, the propagation is not anymore purely azimuthal but has a radial component. The propagation in both directions is quantified by following the center of mass position of the structure through the considered spatial domain. The structure's azimuthal (in angle $\theta$ ) and radial position $r$ for different time lags $\tau$ is shown in Fig. 5. In both directions a constant speed of the structure is found. The azimuthal velocity is $v_{\theta}=270 \mathrm{~m} / \mathrm{s}$, which corresponds to a rotation frequency of the structure around the plasma column of $f=872 \mathrm{~Hz}$. More importantly, a strong radial component of propagation is observed with a radial speed of $v_{r}=160 \mathrm{~m} / \mathrm{s}$, corresponding to $\approx 5 \% C_{s}$, where $C_{s}$ is the ion sound speed at the position of the structure. 


\section{SUMMARY AND CONCLUSION}

The spatiotemporal dynamics of plasma density fluctuations in weakly developed drift wave turbulence in the helicon device VINETA are investigated. Previous studies have unambiguously identified the governing instability in VINETA to be of the drift wave type. By introduction of an axially extended azimuthal limiter we decrease the radial density gradient scale length to drive the drift wave instability into a weakly developed turbulent state. The turbulent density fluctuations have a symmetric PDF in the strong radial plasma density region, while the fluctuation timeseries become burst-like further outwards. The corresponding PDFs become peaked and develop large positive tails. The radial change of intermittency is similar to what has been observed in tokamak SOLs, which is ascribed to radially propagating turbulent structures [17]. The spatiotemporal evolution of coherent fluctuations as analyzed with the conditional averaging technique can be principally distinguished into two spatial regimes. In the strong density gradient region structures resemble very much a $m=1$ drift mode. In particular, it propagates in electron diamagnetic drift direction without any radial component of propagation. In the plasma edge a monopolar structure is found, which propagates azimuthally in $E_{r} \times B$ direction, which is opposite to the mode-like propagation. The density of the structure equals the time-averaged density in the strong gradient region, i.e. in the drift wave unstable region. More importantly, the structure has a radial velocity component, which is determined to be approx. half of its azimuthal velocity and leads to radial displacement during its lifetime, thereby significantly contributing to radial transport.

[1] W. Horton, Rev. Mod. Phys. 71(3), 735 (1999).

[2] J. L. Terry, S. J. Zweben, K. Hallatschek, B. LaBombard, R. J. Maqueda, B. Bai, C. J. Boswell, M. Greenwald, D. Kopon, W. M. Nevins, C. S. Pitcher, B. N. Rogers, et al., Phys. Plasmas 10(5), 1739 (2003).

[3] S. J. Zweben, R. J. Maqueda, D. P. Stotler, A. Keese, J. Boedo, C. E. Bush, S. M. Kaye, B. LeBlanc, J. L. Lowrance, V. J. Mastrocola, R. Maingi, N. Nishino, et al., Nucl. Fusion 44, 134 (2004).

[4] O. Grulke, J. L. Terry, B. LaBombard, and S. J. Zweben, Phys. Plasmas 13, 012306 (2006).

[5] O. E. Garcia, V. Naulin, A. H. Nielsen, and J. J. Rasmussen, Phys. Rev. Lett. 92(16), 165003 (2004).

[6] G. Q. Yu and S. I. Krasheninnikov, Phys. Plasmas 10(11), 4413 (2003).

[7] D. A. D’Ippolito, J. R. Myra, and S. I. Krasheninnikov, Phys. Plasmas 9(1), 222 (2002).

[8] G. Y. Antar, Phys. Plasmas 10(9), 3629 (2003).

[9] T. Carter, Phys. Plasmas 13, 010701 (2006).

[10] C. Schröder, O. Grulke, T. Klinger, and V. Naulin, Phys. Plasmas 11(9), 4249 (2004). 
[11] C. Schröder, O. Grulke, T. Klinger, and V. Naulin, Phys. Plasmas 12(4), 42103 (2005).

[12] C. M. Franck, O. Grulke, and T. Klinger, Phys. Plasmas 9(8), 3254 (2002).

[13] R. W. Boswell, Plasma Phys. Controlled Fusion 26(10), 1147 (1984).

[14] M. Light and F. F. Chen, Phys. Plasmas 2(4), 1084 (1995).

[15] T. Huld, A. H. Nielsen, H. L. Pécseli, and J. J. Rasmussen, Phys. Fluids B 3(7), 1609 (1991).

[16] O. Grulke, T. Klinger, M. Endler, A. Piel, and the W7-AS team, Phys. Plasmas 8(12), 5171 (2001).

[17] O. E. Garcia, J. Horacek, R. A. Pitts, A. H. Nielsen, W. Fundamenski, J. P. Graves, V. Naulin, and J. J. Rasmussen, PLASMA PHYSICS AND CONTROLLED FUSION 48(1), L1 (2006). 
Figure 1: Radial plasma density and plasma potential profiles.

Figure 2: Time series of density fluctuations and respective probability distribution functions at three different radial position (top row $\mathrm{r}=20 \mathrm{~mm}$, middle row $\mathrm{r}=50 \mathrm{~mm}$, bottom row $\mathrm{r}=80 \mathrm{~mm}$ )

Figure 3: Grayscale plot of conditionally averaged density fluctuations (condition $0.5 \sigma$ ) in the azimuthal plane for three different time lags $\tau$ with the reference in the density gradient region $(\mathrm{r}=20 \mathrm{~mm})$. The white circle indicates the maximum density gradient position.

Figure 4: Grayscale plot of conditionally averaged density fluctuations in the same representation as Fig. 3 but with the reference in the plasma edge $(\mathrm{r}=50 \mathrm{~mm})$ and for a condition $3 \sigma$. The white circle indicates the azimuthal direction.

Figure 5: Azimuthal frequency and radial velocity of the structure propagation as determined from the turbulent structure Fig. 4. 


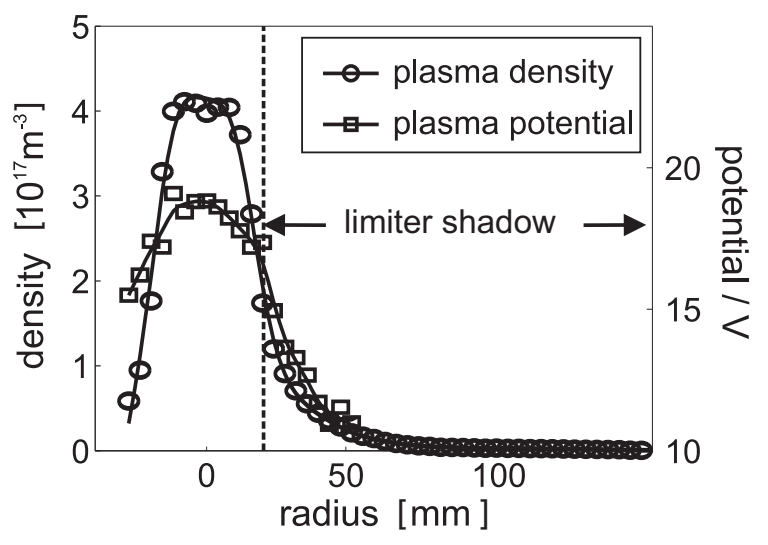

FIG. 1:
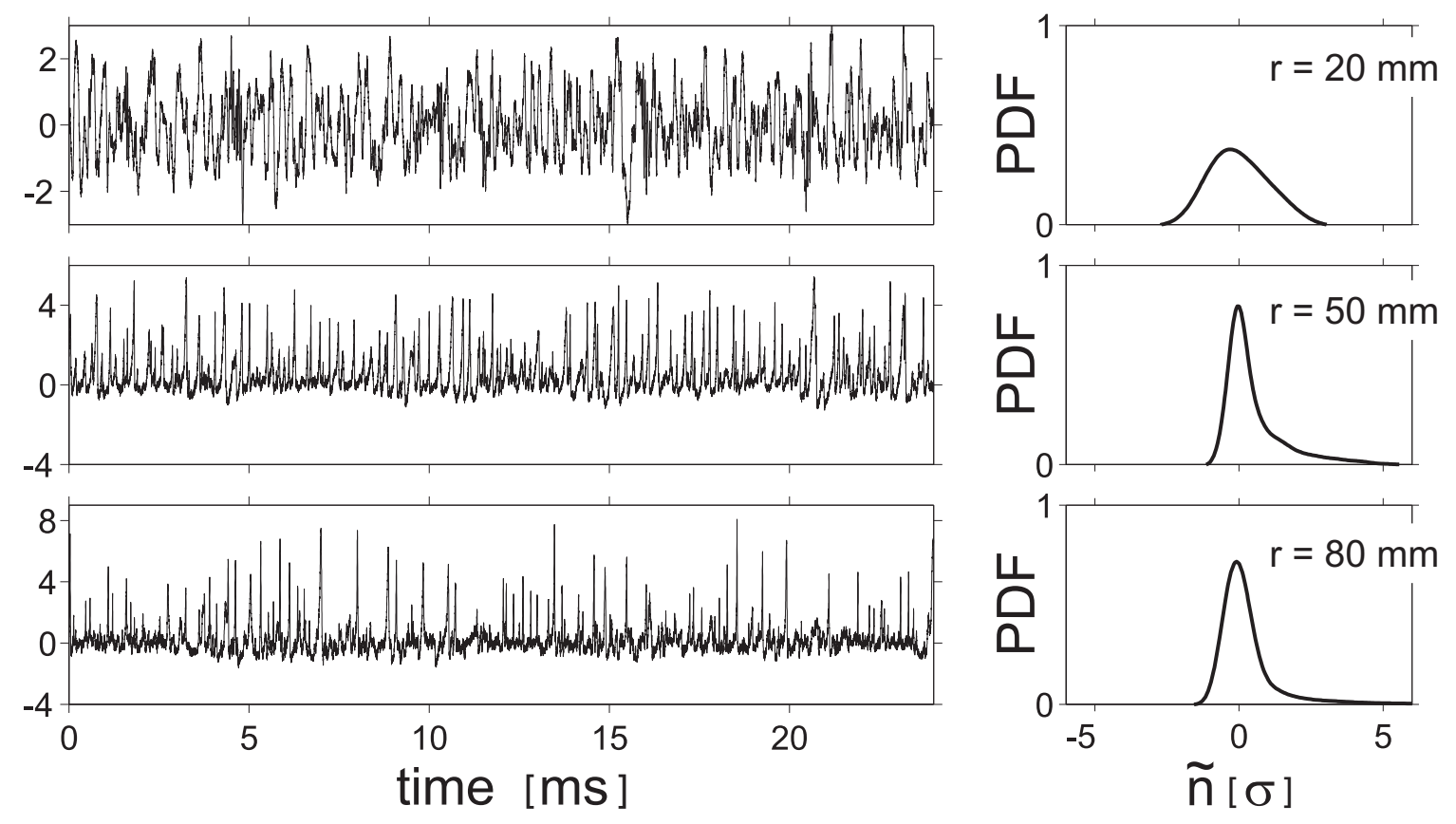

FIG. 2:

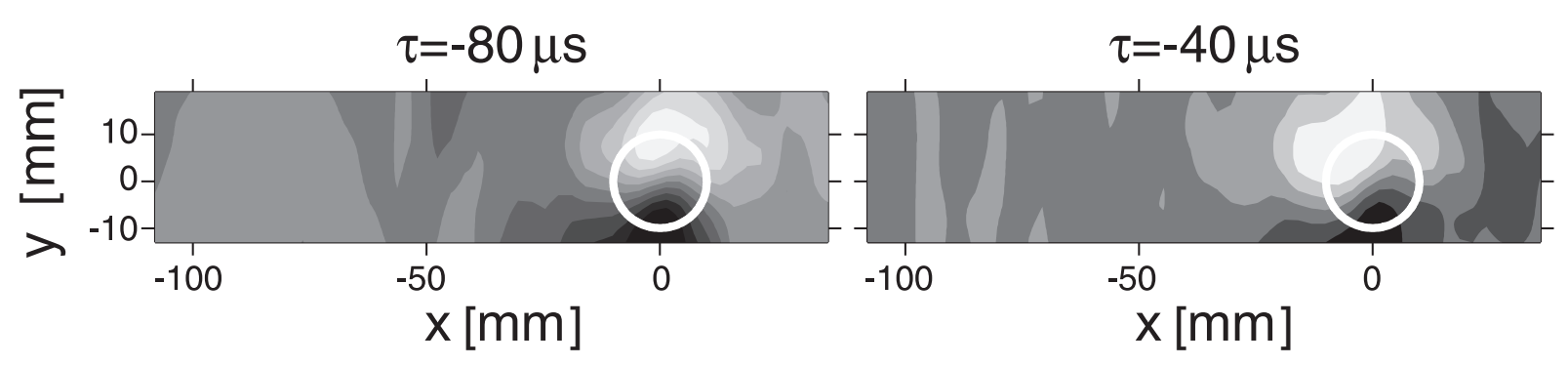

FIG. 3: 

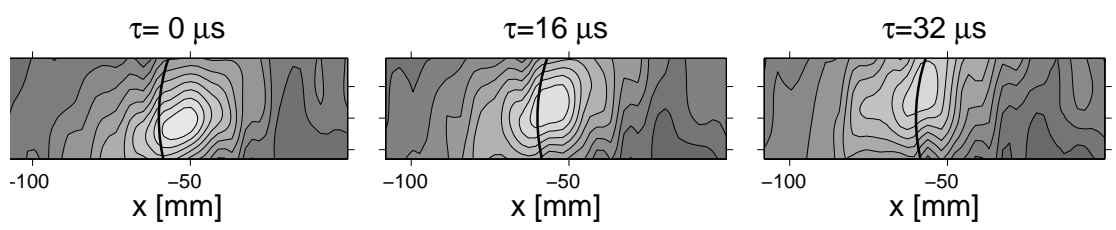

FIG. 4:
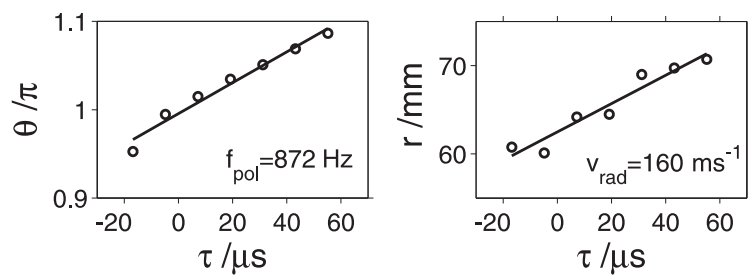

FIG. 5: 